$\Rightarrow$ MECHANISMS OF DISEASE

\title{
Under pressure
}

DOI:

10.1038/nrm1934

\begin{abstract}
A new link between blood-pressure homeostasis and the dysregulation of transforming growth factor- $\beta$ (TGF $\beta$ ) has been reported in Cell. TGF $\beta$ has a key role in the development and pathophysiology of blood vessels, and increased levels of circulating TGF $\beta$ have been reported in hypertensive individuals. TGF $\beta$ and the secreted extracellular matrix (ECM) glycoprotein EMILIN1 (elastin microfibril interface-located protein-1) are co-expressed in vascular smooth-muscle cells of the aortic wall. Based on their analysis of Emilin1-knockout mice, Zacchigna et al. have shown that EMILIN1 is a negative regulator of TGF $\beta$ process-
\end{abstract}

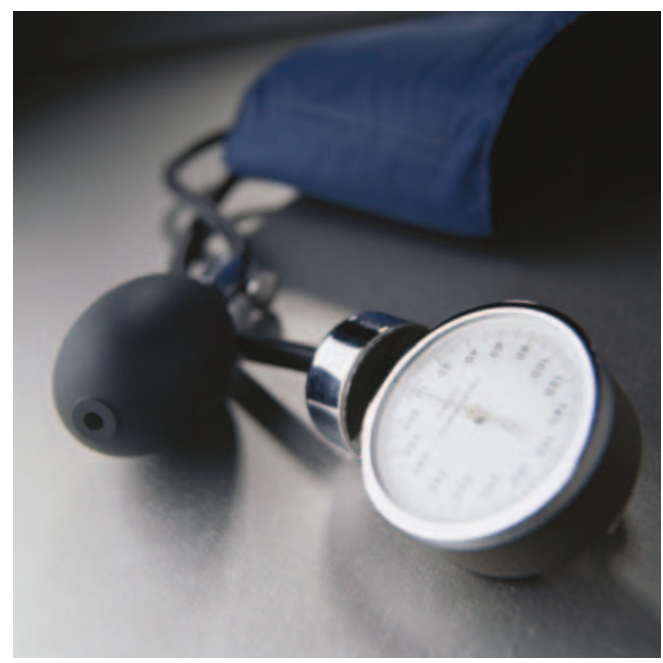

ing and activation, and they propose that this antagonism helps to explain hypertension in animals that lack EMILIN1.

By characterizing the cardiovascular structure and function of Emilin1-null mice, the authors found that blood-vessel diameter was reduced and both peripheral resistance and systemic blood pressure were increased. So what is the mechanism by which EMILIN1 controls vascular-cell behaviour? EMILIN1 is a multidomain protein that includes a cysteine-rich EMI domain. Zacchigna and colleagues reasoned that because other secreted proteins with cysteine-rich repeats are known to regulate growthfactor signalling, it was possible that EMILIN1 might modulate TGF $\beta$ activity in vascular cells through its EMI domain.

Experiments in Xenopus laevis embryos showed that EMILIN1 functions as an antagonist of TGF $\beta$ ligands; further in vitro studies in mammalian cells indicated that the EMI domain binds specifically to proTGF $\beta 1$, the inactive proprotein of TGF $\beta$, in the extracellular space. The precursor was therefore protected from proteolytic activation, and the subsequent production of mature TGF $\beta$ was prevented. But what is the in vivo significance of this interaction? The authors predicted that Emilin1-knockout mice would show increased TGF $\beta$ signalling in the vascular system, and they found supportive evidence by using several assays.

Zacchigna et al. have shown that EMILIN1, a glycoprotein that is associated with the ECM of blood vessels, can modulate TGF $\beta$ signalling, and they have "....identified a new mechanism of regulation of arterial blood pressure.... Their work highlights the importance of the ECM components of the vascular system and the modulation of TGF $\beta$ availability in the pathogenesis of hypertension, and could potentially lead to specific therapies that would be aimed at the origin of the disease. In addition, as the authors point out, their findings could be relevant beyond hypertension, as TGF $\beta$ is thought to have a role in many pathological conditions, including fibrosis, inflammation and cancer.

Sharon Ahmad

ORIGINAL RESEARCH PAPER Zacchigna, L. et al. Emilin 1 links TGF- $\beta$ maturation to blood pressure homeostasis. Cell 124, 929-942 (2006)

FURTHER READING Raman, M. $\&$ Cobb, M. H. TGF- $\beta$ regulation by Emilin1: new links in the etiology of hypertension. Cell 124, 893-895 (2006) 